\title{
Correlations for the ignition delay times of hydrogen/air mixtures
}

\author{
ZHAO ZhenLong, CHEN Zheng ${ }^{*} \&$ CHEN ShiYi \\ State Key Laboratory for Turbulence and Complex Systems, College of Engineering, Peking University, Beijing 100871, China
}

Received November 8, 2009; accepted January 19, 2010

Correlations for the ignition delay times of hydrogen/air mixtures were developed using the method of High Dimensional Model Representation (HDMR). The hydrogen/air ignition delay times for initial conditions over a wide range of temperatures from 800 to $1600 \mathrm{~K}$, pressures from 0.1 to $100 \mathrm{~atm}$, and equivalence ratios from 0.2 to 10 were first calculated utilizing the full chemical mechanism. Correlations were then developed based on these ignition delay times. Two forms of correlations were constructed: the first one is an overall general model covering the whole range of the initial conditions; while the second one is a piecewise correlation model valid for initial conditions within different sub-domains. The performance of these correlations was studied through comparison with results from the full chemical mechanism as well as experimental data. It was shown that these correlations work well over the whole range of initial conditions and that the accuracy can be significantly improved by using different piecewise correlations for different sub-domains. Therefore, piecewise correlations can be used as an effective replacement for the full mechanism when the prediction of chemical time scale is needed in certain combustion modeling.

hydrogen/air, ignition delay time, correlation, HDMR

Citation: Zhao Z L, Chen Z, Chen S Y. Correlations for the ignition delay times of hydrogen/air mixtures. Chinese Sci Bull, 2011, 56: 215-221, doi: 10.1007/ s11434-010-4345-3

Ignition delay time or reaction time [1-3] is one of the most important parameters characterizing the combustion process of different fuels and thus is very important for combustion modeling. Accurate calculation of the ignition delay time, especially for the combustion process with a variety of fuel blends in gas turbines and other combustion engines, is a complicated procedure because of the complex interaction between flows and chemical kinetics [4]. For hydrocarbon and synthetic bio-fuels with a large number of species and a broad range of time scales, enormous computation time is required to handle the detailed chemistry in reactive flow simulation [5]. Moreover, it is required to couple a full chemistry solver with a full-scale numerical flow solver which makes the whole procedure time-consuming and far beyond the limitation of current computers [3]. Therefore, there is an increasing need to find simplified alternatives which can accurately and efficiently predict the over-all behavior of all chemical reactions.

*Corresponding author (email: cz@pku.edu.cn)
In order to reduce the computational time due to complex chemistry, different correlations were developed to calculate the ignition delay times for different mixtures. For example, Molnar and Marek [6,7] developed reduced equations from fitted kinetics modeling for jet-A and methane fuels. More recently, Donato and Petersen [3] provided simplified correlation equations to calculate the ignition delay times of syngas/air mixtures at different initial temperatures, pressures, and equivalence ratios. These correlations $[3,6,7]$ were obtained through curve fitting and thus the accuracy was highly constrained by the nonlinear regression process. Instead of conducting nonlinear regression, the method of High Dimensional Model Representation (HDMR, to be introduced in the next section) [8-12] is utilized in this paper to give more accurate correlations for the ignition delay times. Since hydrogen is widely considered as a candidate for the next-generation energy carrier, the ignition of hydrogen is important not only for combustion engines but also for safe transport and utilization of hydrogen. Therefore, correlations for the ignition delay times of 
hydrogen/air mixtures are developed in this study by using the HDMR method.

\section{HDMR method}

The High Dimensional Model Representation (HDMR) method is a set of tools developed by Rabitz et al. [8-12] to capture high-dimensional input-output relationship of a complex model with a large number of input parameters. It expresses the output variable $f(\boldsymbol{x})$ as a finite hierarchical correlated function expansion in terms of the input variables $\boldsymbol{x}=\left(x_{1}, x_{2}, \ldots, x_{n}\right)$ in the following form [8-12]:

$$
\begin{aligned}
f(\boldsymbol{x})= & f_{0}+\sum_{i=1}^{n} f_{i}\left(x_{i}\right)+\sum_{1 \leqslant i<j \leqslant n} f_{i j}\left(x_{i}, x_{j}\right)+\cdots \\
& +f_{1,2, \cdots, n}\left(x_{1}, x_{2}, \ldots, x_{n}\right),
\end{aligned}
$$

where the zeroth-order component function $f_{0}$ is a constant denoting the mean response: the first-order component function $f_{i}\left(x_{i}\right)$ gives the individual contribution to the output $f(\boldsymbol{x})$ acted by the $i$ th input variable; the second-order function $f_{i j}\left(x_{i}, x_{j}\right)$ describes the cooperative effects of the input variables $x_{i}$ and $x_{j}$ upon the output $f(\boldsymbol{x})$, etc. The last term $f_{1,2, \ldots, n}\left(x_{1}, x_{2}, \ldots, x_{n}\right)$ reflects any residual $n$ th-order correlated contribution of all input variables to the output $f(\boldsymbol{x})$.

Systematic optimal procedures have been developed to construct the distinct HDMR component functions in the above expansion [8-12]. Recently, random sampling (RS)-HDMR has been developed by Li et al. [8] to accurately and efficiently treat high dimensional input-output mapping problems and has been successfully utilized in several scientific modeling applications including atmospheric chemistry [11], molecular dynamics simulations [8], and bio-kinetics modeling [13]. Therefore, RS-HDMR is utilized in this study to construct correlations for the ignition delay times of hydrogen/air mixtures.

To reduce the sampling effort, the RS-HDMR component functions are approximated by optimal weighted orthonormal polynomials $\{\varphi\}$ as [8]

$$
\begin{aligned}
& f_{i}\left(x_{i}\right) \approx \sum_{r=1}^{k} \alpha_{r}^{(0) i} \varphi_{r}^{i}\left(x_{i}\right), \\
& f_{i j}\left(x_{i}, x_{j}\right) \approx \sum_{r=1}^{k}\left[\alpha_{r}^{(i j) i} \varphi_{r}^{i}\left(x_{i}\right)+\alpha_{r}^{(i j) j} \varphi_{r}^{j}\left(x_{j}\right)\right] \\
& +\sum_{p=1}^{l} \sum_{q=1}^{l^{\prime}} \beta_{p q}^{(0) i j} \varphi_{p}^{i}\left(x_{i}\right) \varphi_{q}^{j}\left(x_{j}\right), \\
& \left.\quad+\alpha_{r}^{(i j k) k} \varphi_{r}^{k}\left(x_{k}\right)\right]+\sum_{p=1}^{l} \sum_{q=1}^{l^{\prime}}\left[\beta_{p q}^{(i j k) i j} \varphi_{p}^{i}\left(x_{i}\right) \varphi_{q}^{j}\left(x_{j}\right),\right. \\
& \left.\quad+\beta_{p q}^{(i j k) i k} \varphi_{p}^{i}\left(x_{i}\right) \varphi_{q}^{k}\left(x_{k}\right)+\beta_{p q}^{(i j k) j k} \varphi_{p}^{j}\left(x_{j}\right) \varphi_{q}^{k}\left(x_{k}\right)\right]
\end{aligned}
$$

$$
+\sum_{p=1}^{m} \sum_{q=1}^{m^{\prime}} \sum_{r=1}^{m^{\prime \prime}} \gamma_{p q r}^{(0) i j k} \varphi_{p}^{i}\left(x_{i}\right) \varphi_{q}^{j}\left(x_{j}\right) \varphi_{r}^{k}\left(x_{k}\right),
$$

where $K, l, l^{\prime}, m, m^{\prime}, m^{\prime \prime}$ are integers, $\alpha_{r}, \beta_{p q}$, and $\gamma_{p q r}$ are constant coefficients to be determined and $\{\varphi\}$ are defined as [8]

$$
\left\{\begin{array}{c}
\phi_{1}^{i}\left(x_{i}\right)=a_{1} x_{i}+a_{0}, \\
\phi_{2}^{i}\left(x_{i}\right)=b_{2} x_{i}^{2}+b_{1} x_{i}+b_{0}, \\
\phi_{3}^{i}\left(x_{i}\right)=c_{3} x_{i}^{3}+c_{2} x_{i}^{2}+c_{1} x_{i}+c_{0} \\
\cdots
\end{array}\right.
$$

The constant coefficients $a_{0}, a_{1}, b_{0}, \ldots, c_{3}$ are determined to ensure the orthonormality of $\{\varphi\}$ for a given set of random samples. The details on how to construct the RSHDMR component functions can be found in [8] and thus are not repeated here.

It was noted that only lower order component functions have significant contributions to the output since the HDMR component functions are optimally constructed [8,10,11]. Therefore third-order HDMR is accurate enough and is used here.

\section{Results and discussion}

In this study, the output is the ignition delay time, $\tau_{i g}$, of a homogeneous hydrogen/air mixture. The detailed hydrogen/air mechanism recently developed by Li et al. [14] was used in parallel with the SENKIN program of CHEMKIN $[15,16]$ to simulate the homogeneous ignition process. The calculations were performed assuming the constant enthalpy and constant pressure due to the combustor applications of the ignition delay times. The ignition delay time of constant-pressure ignition is close to that of constant-volume ignition. Therefore, the results herein can also be used for constant-volume applications.

There are many different ways to define the ignition delay time. In this study, the definition of ignition delay time is based on the evolution of the temperature as a function of time, $T(t)$, and $\tau_{i g}$ is the time when the largest temperature changing rate $(\mathrm{d} T / \mathrm{d} t)$ occurs. Figure 1 shows a typical result of temperature plotted against time and the ignition delay time according to this definition is $\tau_{i g}=44.9 \mu \mathrm{s}$. It is also acceptable to define the ignition delay time as the time when the temperature reaches certain degree above the initial temperature, e.g. $T+\Delta T$, or as the sudden rise in $\mathrm{OH}^{*}$ concentration, where $\mathrm{OH}^{*}$ represents the excited electronic state of $\mathrm{OH}$ as commonly used in shock-tube experiments [17]. As shown in Figure 1, the ignition delay times determined in these three ways are very close (the relative difference is shown to be less than $1.5 \%$ ). Therefore, different definitions of the ignition delay times can be used interchangeably since they are quantitatively similar. For other cases within the range of mixtures and initial conditions (temperature and pressure), 


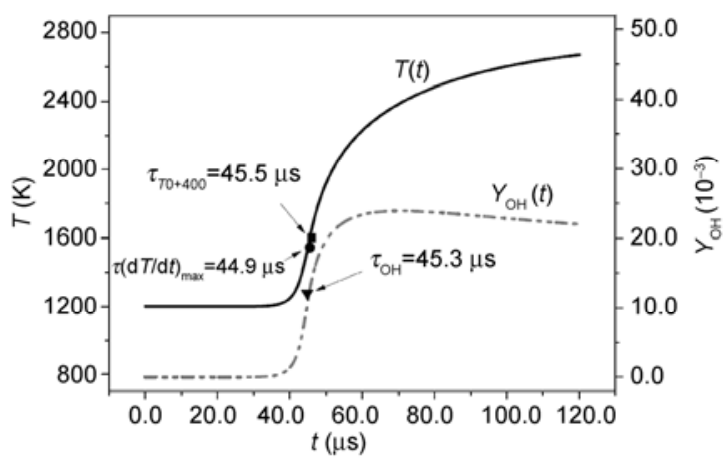

Figure 1 Evolution of temperature and mass fraction of $\mathrm{OH}$ radical during the homogenous ignition of a stoichiometric hydrogen/air mixture initially at $1200 \mathrm{~K}$ and $1 \mathrm{~atm}$. Three definitions of the ignition delay time are also shown.

the conclusion remains the same.

In order to construct the correlations for the ignition delay times of hydrogen/air mixtures, different ignition delay times at different initial temperatures, $T_{0}$, pressures, $P$, and equivalence ratios, $\phi$, should be calculated first, and then these results are utilized as samples to construct one (or more) correlation(s) via RS-HDMR. It is well known that the correlation for the ignition delay time is in the form of Arrhenius-type formula [18]

$$
\tau_{i g} \propto \phi^{x} P^{y} \exp \left(\frac{E}{R T}\right) \text {. }
$$

Therefore, the following correlation(s) are constructed via RS-HDMR method

$$
\log _{10} \tau_{\text {ig }}=f\left(1 / T_{0}, \log _{10} P, \log _{10} \phi\right) .
$$

The inputs, i.e. $\left(1 / T_{0}, \log _{10} P, \log _{10} \phi\right)$, are randomly sampled with a uniform distribution within the domain of $1 / 1600<1 /\left(T_{0}\right)<1 / 800,-1<\log _{10}(P)<2$, and $\log _{10} 0.2<\log _{10} \phi<1$. Figure 2 shows the ignition delay times predicted by SENKIN as a function of the initial temperature and pressure. Results from 2000 computer-runs with different initial conditions are presented. It is seen that at a given pressure, the ignition delay time monotonically increases with the inverse initial temperature. With the increase of $1 / T_{0}$, an abrupt change of the slope between $\log _{10}\left(\tau_{i g}\right)$ and $1 / T_{0}$ is shown to appear at some transition temperature, $T_{0}{ }^{*}$. The slope at the higher-temperature regime $\left(1 / T_{0}<1 / T_{0}{ }^{*}\right)$ is shown to be much smaller than that at the lowertemperature regime $\left(1 / T_{0}>1 / T_{0}{ }^{*}\right)$. Moreover, Figure 2 also shows that the transition temperature monotonically increases with the pressure. For a given initial temperature, it is found (also shown in Figure 6) that the ignition delay time changes non-monotonically with the pressure. This is because hydrogen oxidation is controlled by the competition between the branching reactions, $\mathrm{H}+\mathrm{O}_{2}=\mathrm{O}+\mathrm{OH}$ and $\mathrm{H}_{2} \mathrm{O}_{2}+\mathrm{M}=\mathrm{OH}+\mathrm{OH}+\mathrm{M}$, and the termination reaction, $\mathrm{H}+\mathrm{O}_{2}+$ $\mathrm{M}=\mathrm{HO}_{2}+\mathrm{M}$, the reaction rates of which strongly depend on

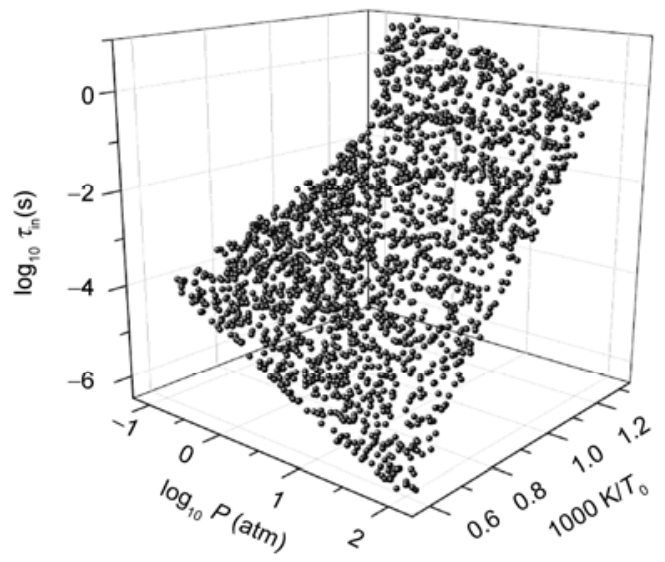

Figure 2 Ignition delay time as a function of initial temperature and pressure.

the pressure [18]. Compared to the initial temperature and pressure, the equivalence ratio is found to exert less influence on the ignition delay time.

The original goal of this study was to obtain a single overall HDMR correlation that would accurately fit the entire set of ignition delay time data. However, the accuracy of the overall HDMR correlation is constrained by the strong non-linearity between the input and output variables, as shown in Figure 2. Therefore, in order to improve the accuracy, a piecewise HDMR correlation model was also proposed.

\subsection{Fitting data to an overall correlation}

The RS-HDMR technique was utilized here to predict the ignition delay times of hydrogen/air mixtures according to eq.(5). 2000 random data points were computed via SENKIN with the initial conditions uniformly distributed within the domain of $1 / 1600<1 /\left(T_{0}\right)<1 / 800,-1<\log _{10}(P)<2$, and $\log _{10} 0.2<\log _{10} \phi<1$. For the entire data set $(M=2000)$, different sample sizes $(N=200,400,600,800,1000$, referred as "used data") were used to construct the overall correlation via RS-HDMR. The remaining $M-N$ data points (referred as "test data") were used to test the performance of the overall correlation. It was found that the performance of the resulting HDMR correlation is not strongly affected by the sample size, $N$, and thus a small sample can be used [8]. Figure 3 compares the ignition delay times predicted by the overall correlation $\left(\tau_{\mathrm{HDMR}}\right)$ and those by SENKIN using the detailed hydrogen/air chemistry $\left(\tau_{\text {Mech }}\right)$. The coefficient of determination, $R^{2}$, is shown to be 0.98 . From this point, the overall correlation predicts the ignition delay time quite well. However, as will be shown later in Figures 5 and 6 , the transition between the higher-and lower-temperature/ pressure regimes is not accurately predicted by the overall correlation, especially for initial conditions at low temperatures and low pressures. In order to improve the accuracy, the whole domain of the initial conditions (temperature and 


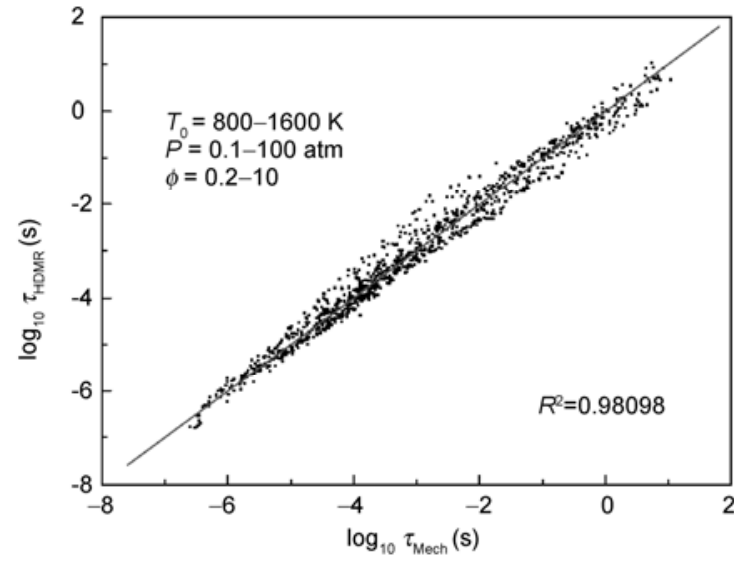

Figure 3 Comparison of the ignition delay times predicted by the overall correlation $\left(\tau_{\mathrm{HDMR}}\right)$ and those by SENKIN using the detailed chemical mechanism $\left(\tau_{\text {Mech }}\right)$.

pressure) needs to be separated into several sub-domains and different correlations should be generated based on the data in each sub-domain.

\subsection{Fitting data to six piecewise correlations}

Due to the strong effects of the initial temperature on the ignition delay time over the full range of parameters in this study, different correlations were developed to describe the higher-temperature and the lower-temperature regimes. Moreover, it is noted that the transition temperature strongly depends on the pressure. Therefore the whole pressure domain ranging from 0.1 to $100 \mathrm{~atm}$ is also divided into several different regions. Many different ways of division were tried. The six-subdomain division summarized in Table 1 was found to be better than others and thus was employed in this study.

For each sub-domain, 2000 random data points were computed via SENKIN with the initial conditions uniformly distributed in terms of $1 / T_{0}, \log _{10} P$ and $\log _{10} \phi$. The first 1000 data were used to construct the correlation via RS-HDMR while the remaining 1000 data were used to test the performance of the piecewise correlation. Compared to the overall correlation which is suitable for the whole domain of the initial conditions (temperature and pressure), the correlation in each sub-domain was called the piecewise correlation. Figure 4 compares the ignition delay times predicted by the piecewise correlation $\left(\tau_{\mathrm{HDMR}}\right)$ and those predicted by SENKIN using the detailed hydrogen/air chemistry $\left(\tau_{\text {Mech }}\right)$ for initial conditions within three different sub-domains, II, III, and V, as listed in Table 1. The coefficients of determination, $R^{2}$, are shown to be close to unity. Therefore the piecewise correlation can accurately predict the ignition delay times at different initial conditions. To compare the performance of the overall and piecewise correlations, the coefficients of determination, $R^{2}$, for different sub-domains are listed in Table 2. As expected, the $R^{2}$ of the piecewise correlation is closer to unity than that of the overall correlation. Therefore, the accuracy can be improved by using the piecewise correlations for sub-domains instead of the overall correlation for the whole domain of the initial conditions (temperature and pressure).

Figure 5 shows the ignition delay time as a function of the initial temperature for stoichiometric hydrogen/air mixtures at different pressures. The results (solid lines) predicted by SENKIN using the detailed chemical mechanism are the exact ignition delay times and are shown here for comparison. At the atmospheric pressure, Figure 5(a) shows that there is a large difference between results from the piecewise and overall correlations, especially for the initial

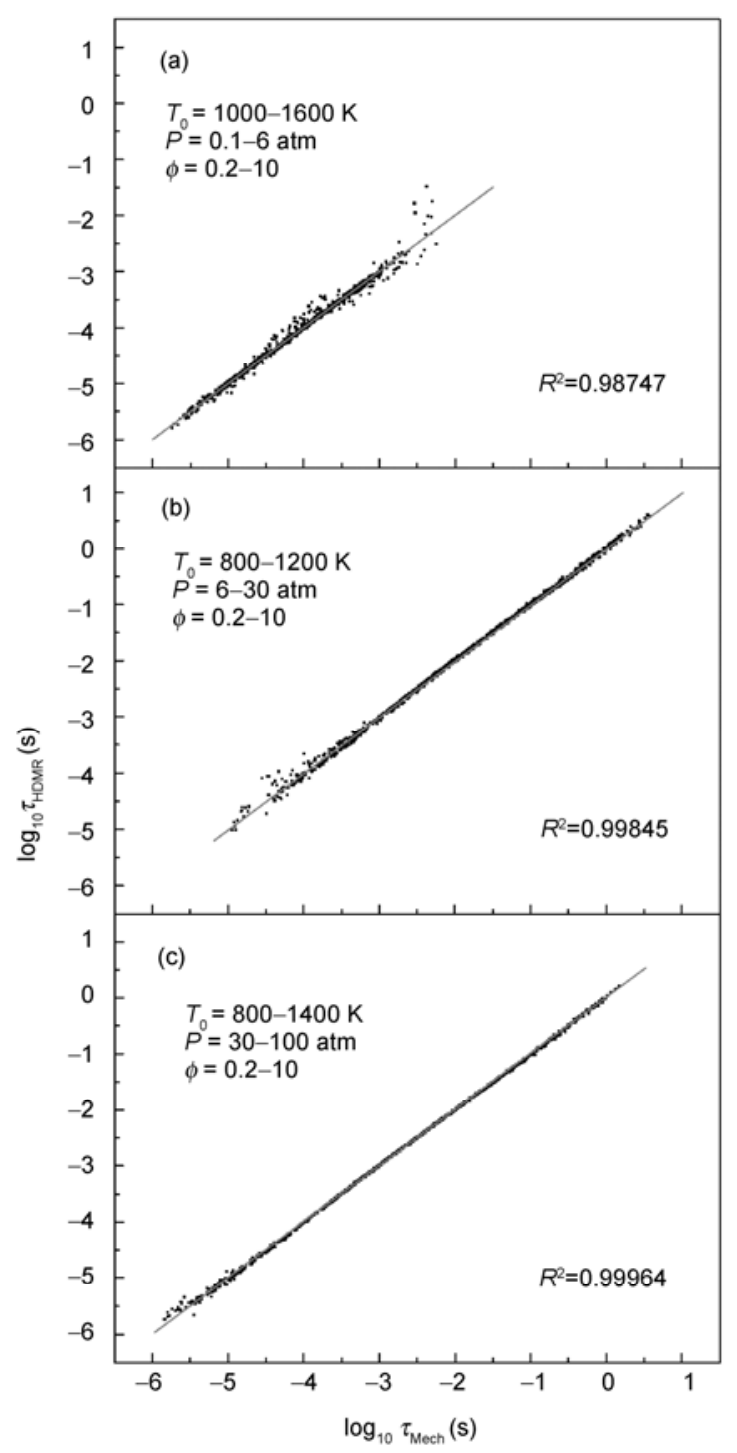

Figure 4 Comparison of the ignition delay times predicted by the piecewise correlation $\left(\tau_{\mathrm{HDMR}}\right)$ and those by SENKIN using the detailed chemical mechanism $\left(\tau_{\text {Mech }}\right)$ for initial conditions within three different sub-domains. (a) Sub-domain II; (b) sub-domain III; (c) sub-domain V. 
Table 1 The initial temperature and pressure ranges for six sub-domains

\begin{tabular}{|c|c|c|c|}
\hline & Low pressure & Intermediate pressure & High pressure \\
\hline Low temperature & $\begin{array}{c}\text { Sub-domain I } \\
800 \leqslant T_{0} \leqslant 1000 \mathrm{~K} \\
0.1 \leqslant P \leqslant 6.0 \text { atm }\end{array}$ & $\begin{array}{c}\text { Sub-domain III } \\
800 \leqslant T_{0} \leqslant 1200 \mathrm{~K} \\
6.0<P \leqslant 30.0 \mathrm{~atm}\end{array}$ & $\begin{array}{c}\text { Sub-domain V } \\
800 \leqslant T_{0} \leqslant 1400 \mathrm{~K} \\
30.0<P \leqslant 100.0 \mathrm{~atm}\end{array}$ \\
\hline High temperature & $\begin{array}{c}\text { Sub-domain II } \\
1000<T_{0} \leqslant 1600 \mathrm{~K} \\
0.1 \leqslant P \leqslant 6.0 \mathrm{~atm}\end{array}$ & $\begin{array}{c}\text { Sub-domain IV } \\
1200<T_{0} \leqslant 1600 \mathrm{~K} \\
6.0<P \leqslant 30.0 \mathrm{~atm}\end{array}$ & $\begin{array}{c}\text { Sub-domain VI } \\
1400<T_{0} \leqslant 1600 \mathrm{~K} \\
30.0<P \leqslant 100.0 \mathrm{~atm}\end{array}$ \\
\hline
\end{tabular}

Table 2 The coefficients of determination, $R^{2}$, for different sub-domains

\begin{tabular}{ccccccc}
\hline Sub-domain & I & II & III & IV & V & VI \\
\hline $\begin{array}{c}R^{2} \text { (overall } \\
\text { correlation) }\end{array}$ & 0.91181 & 0.91999 & 0.97947 & 0.90263 & 0.99157 & 0.81088 \\
$\begin{array}{c}R^{2} \text { (piecewise } \\
\text { correlation) }\end{array}$ & 0.95926 & 0.98747 & 0.99845 & 0.98815 & 0.99964 & 0.98993 \\
\hline
\end{tabular}

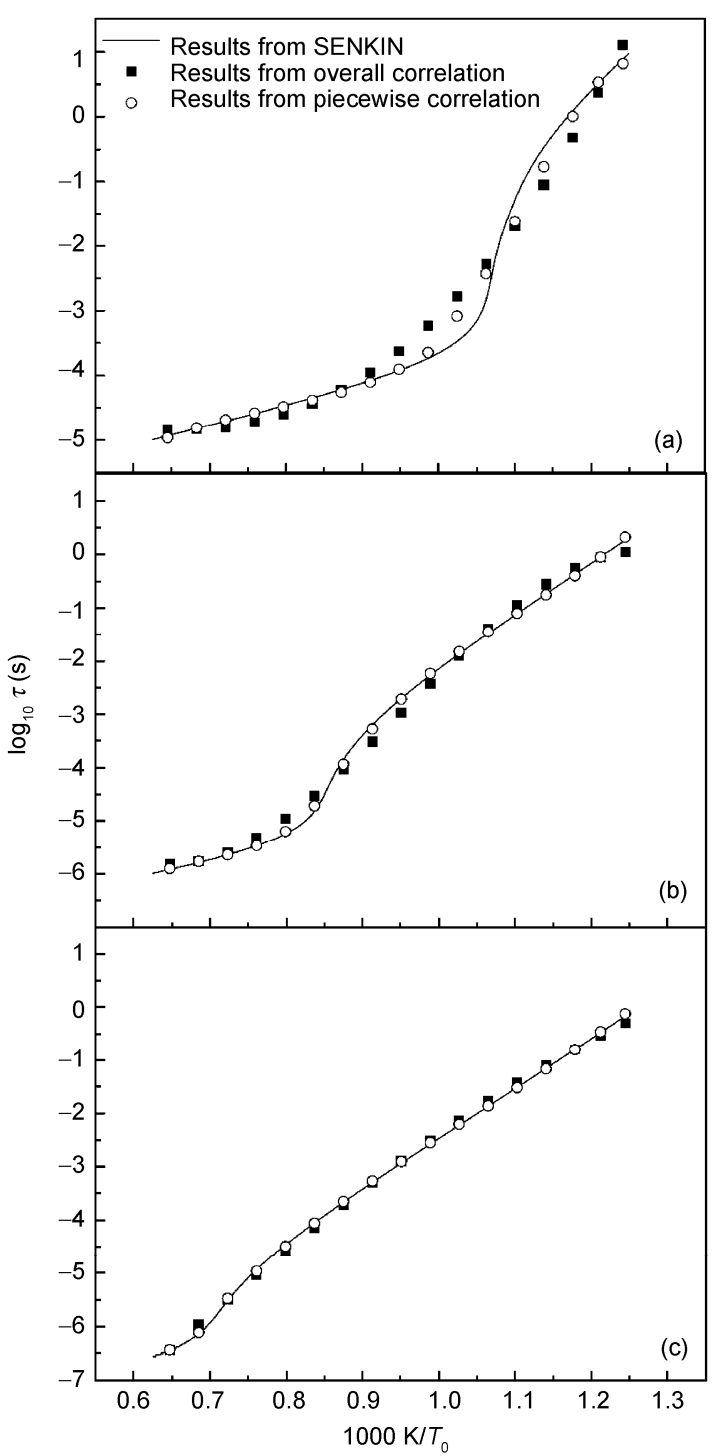

Figure 5 Ignition delay time as a function of the initial temperature for stoichiometric hydrogen/air mixtures at different pressures. (a) $P=1 \mathrm{~atm}$; (b) $P=10$ atm; (c) $P=50$ atm.

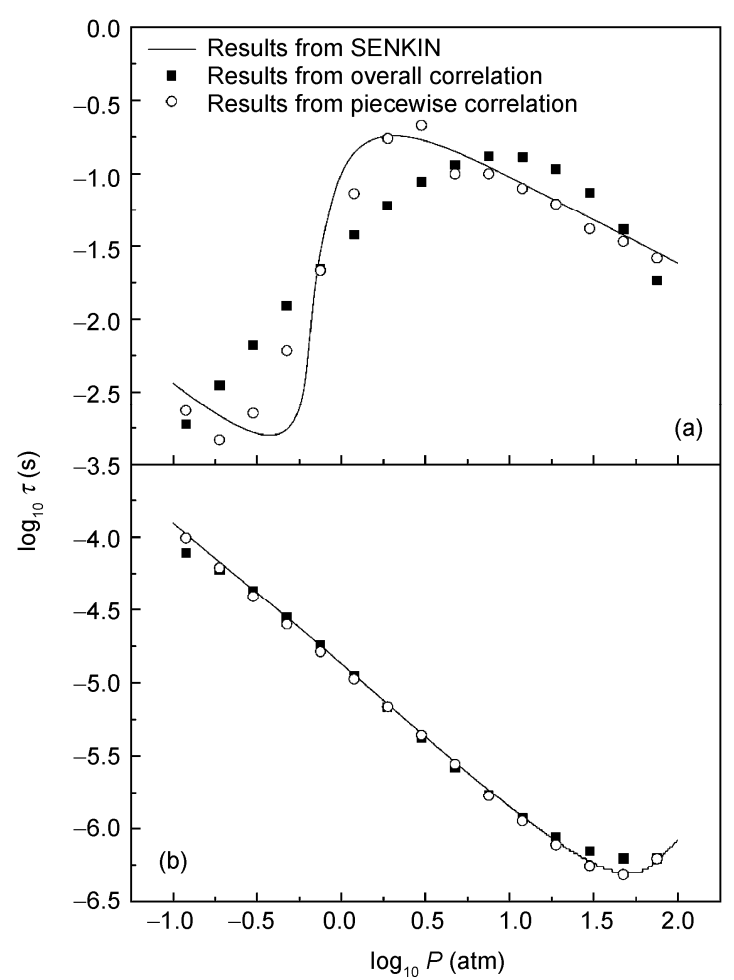

Figure 6 Ignition delay time as a function of pressure for stoichiometric hydrogen/air mixtures at different initial temperatures. (a) $T_{0}=900 \mathrm{~K}$; (b) $T_{0}=1500 \mathrm{~K}$.

temperature close to the transition temperature, $T_{0}{ }^{*}$ (around $900 \mathrm{~K}$ for $P=1 \mathrm{~atm})$. It is seen that the overall correlation cannot accurately predict the ignition delay time for the initial temperature around the transition temperature and within the lower-temperature regime. By using two different piecewise correlations for the higher-and lower-temperature regimes, respectively, Figure 5(a) shows that the accuracy is greatly improved. At intermediate pressure $(P=10 \mathrm{~atm})$ and high pressure $(P=50 \mathrm{~atm})$, Figure $5(\mathrm{~b})$ and $5(\mathrm{c})$ show that the results from the piecewise and overall correlations are very close, both of which agree well with the exact results from SENKIN. Compared to the exact ignition delay times (solid line), results from the piecewise correlations are shown to be more accurate than those from the overall correlation.

Similarly, Figure 6 shows the ignition delay time as a function of pressure for stoichiometric hydrogen/air mixtures at different temperatures. At the lower initial temperature $\left(T_{0}=900 \mathrm{~K}\right)$, Figure 6 (a) shows that the ignition delay 
time changes non-monotonically with the pressure: it first decreases, then increases, and finally decreases again with the pressure. As mentioned before, this non-linear behavior is due to the fact that the reaction rates of the branching reactions, $\mathrm{H}+\mathrm{O}_{2}=\mathrm{O}+\mathrm{OH}$ and $\mathrm{H}_{2} \mathrm{O}_{2}+\mathrm{M}=\mathrm{OH}+\mathrm{OH}+\mathrm{M}$, and the termination reaction, $\mathrm{H}+\mathrm{O}_{2}+\mathrm{M}=\mathrm{HO}_{2}+\mathrm{M}$, strongly depend on the pressure [18]. It is seen that the overall correlation cannot even qualitatively predict the non-linear behavior: compared to the exact results predicted by SENKIN using the detailed chemical mechanism (solid line), the opposite trend at which the ignition delay time changes with the pressure in lower pressure regime $\left(\log _{10} P<-0.4\right)$ is predicted by the overall correlation. By using three different piecewise correlations for the low-, intermediate-, and highpressure regimes, respectively, the non-linear behavior is captured and the accuracy is greatly improved. Figure 6(a) shows that the piecewise correlations can accurately predict the ignition delay times at high pressures while large discrepancies between the exact results and those from piecewise correlations still exist for low-and intermediate- pressure regimes. Unlike the lower temperature $\left(T_{0}=900 \mathrm{~K}\right)$ results, Figure 6(b) shows that for the higher initial temperature $\left(T_{0}=1500 \mathrm{~K}\right)$, results from the piecewise and overall correlations both agree well with the exact results from SENKIN. As expected, the piecewise correlations are also shown to be more accurate than the overall correlation, especially for the high-pressure regime in which the nonlinear behavior occurs.

\subsection{Discussion}

In this study, a wide range of conditions including temperatures from 800 to $1600 \mathrm{~K}$, pressures from 0.1 to $100 \mathrm{~atm}$, and equivalence ratios from 0.2 to 10.0 is covered to construct the correlations for the ignition delay times of hydrogen/air mixtures. To the authors' knowledge, this is the first attempt to use the HDMR method to develop correlations for hydrogen/air mixtures covering such an extensive range of conditions. Compared to the work done by Molnar et al. [6,7] and Donato et al. [3], which focus on developing reduced equations for the specific chemical mechanisms, the correlations constructed in this study are much more accurate with the help of HDMR technique.

As shown in the above sections, two forms of correlations, overall one and piecewise one, are constructed to represent the ignition delay times as a function of initial temperature, pressure, and equivalence ratio. The overall correlation uses a single equation to predict the ignition delay time. The advantage of the overall correlation is that only one group of coefficients needs to be evaluated for the wide range of initial conditions. However, the accuracy of the overall correlation is sacrificed by its simplicity. It can model the higher- and lower-pressure, higher- and lowertemperature regimes; however the transition parts between these regimes cannot be accurately predicted. For the piecewise correlations, different equations are utilized to predict the ignition delay times for the initial conditions within different regimes. The accuracy is shown to be greatly improved by replacing the overall correlation with the piecewise correlations. For the initial temperature close to the transition temperature, $T_{0}{ }^{*}$, it is seen that the overall correlation cannot accurately predict the ignition delay time. By using two different piecewise correlations for the higherand lower-temperature regimes, respectively, the ignition delay time for the initial temperature close to the transition temperature is found to be accurately predicted.

To further demonstrate the performance of the overall and piecewise correlations, Figure 7 compares the ignition delay times of hydrogen/air mixtures measured experimentally by shock tubes $[19,20]$ and those predicted by different correlations developed in this study. It is seen that both the overall and piecewise correlations correctly predict the tendency of the ignition delay time changing with the inverse initial temperature. The piecewise correlations are shown to give much better results than the overall correlations. For $P=2.5$ atm, predictions from the piecewise correlations are almost the same as the experimental results. For $P=2$ atm, the experimental results are slightly lower than those from piecewise correlations but the difference is still very small considering the large uncertainty in the ignition delay times. Therefore, the piecewise correlations can accurately predict the ignition delay times of hydrogen/air mixtures and thus can be used in combustion modeling replacing full chemical mechanisms.

\section{Conclusions}

The method of HDMR (High Dimensional Model Representation) was utilized to construct correlations for the hydrogen/ air ignition delay times over a wide range of initial conditions. Two forms of HDMR correlations were constructed: the first one is an overall general model covering the whole range of the initial conditions, while the second one is a

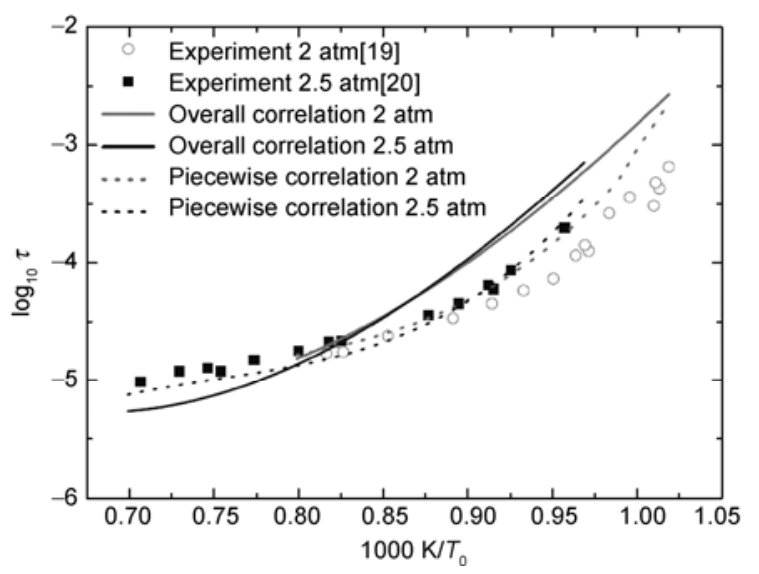

Figure 7 Comparison of the ignition delay time of stoichiometric hydrogen/air mixtures measured from experiments (symbols) and predicted by HDMR correlations (lines) 
piecewise correlation model valid for initial conditions within different sub-domains. The overall HDMR correlation gives a good prediction of the behaviors of the ignition delay times but cannot accurately model the transition parts between the higher- and lower-pressure, high-and lowtemperature regimes. By constructing different piecewise HDMR correlations for different sub-domains, the accuracy can be significantly improved and the piecewise correlations were shown to be able to accurately predict the ignition delay times of hydrogen/air mixtures, even at transition parts. Therefore, HDMR correlations can be used as an effective replacement for the full mechanism when the prediction of chemical time scale is needed in certain combustion modeling. These HDMR correlations are available from the authors on request.

This work was partially supported by National Natural Science Foundation of China (50976003), Beijing Natural Science Foundation (3102016), and discretionary research fund from State Key Laboratory for Turbulence and Complex Systems. Zheng Chen would like to thank Dr. Gengyuan Li at Princeton University for helpful discussions.

1 Westbrook C K, Dryer F L. Simplified reaction mechanisms for the oxidation of hydrocarbon fuels in flames. Combust Sci Tech, 1981, 27: $31-43$

2 Clifford L J, Milne A M, Murray B A. Numerical modeling of chemistry and gas dynamics during shock-induced ethylene combustion. Combust Flame, 1996, 104: 311-327

3 Donato N S, Petersen E L. Simplified correlation models for $\mathrm{CO} / \mathrm{H}_{2}$ chemical reaction times. Int J Hydrogen Energy, 2008, 33: 75657579

4 Lieuwen T, McDonell V, Petersen E, et al. Fuel flexibility influences on premixed combustor blowout, flashback, autoignition, and stability. Eng Gas Turbines Power, 2008, 130: 011506

5 Law C K, Lu T. Towards accommodating realistic fuel chemistry in large-scale computations. AIAA 2008-969, 2008
6 Molnar M, Marek C J. Reduced equations for calculating the combustion rates of jet-A and methane fuel. NASA, TM-212702, 2003

7 Molnar M, Marek C J. Simplified two-time step method for calculating combustion and emission rates of jet-A and methane fuel with and without water injection. AIAA paper 2005-0549, 2005

8 Li G Y, Hu J S, Wang S W, et al. Random sampling-high dimensional model representation (RS-HDMR) and orthogonality of its different order component functions. J Phys Chem A, 2006, 110: 2474-2485

9 Rabitz H, Alis O F, Shorter J, et al. Efficient input-output model representations. Comput Phys Commun, 1999, 117: 11-20

10 Shorter J, Ip P C, Rabitz H. An efficient chemical kinetics solver using high dimensional model representation. J Phys Chem A, 1999, 103: 7192-7198

11 Li G Y, Wang S W, Rabitz H. Practical approaches to construct RS-HDMR component functions. J Phys Chem A, 2002, 106: 87218733

12 Li G Y, Rabitz H, Hu J S, et al. Regularized random-sampling high dimensional model representation (RS-HDMR). J Math Chem, 2008; 43: $1207-1232$

13 Feng X J, Hooshangi S, Chen D, et al. Optimizing genetic Circuits by global sensitivity analysis. Biophys J, 2004, 87: 2195-2202

14 Li J, Zhao Z, Kazakov A, et al. An updated comprehensive kinetic model of hydrogen combustion. Int J Chem Kinet, 2004, 36: 566-575

15 Lutz A, Kee R, Miller J. SENKIN: A FORTRAN program for predicting homogeneous gas phase chemical kinetics with sensitivity analysis. Sandia Report SAND87-8248, Sandia National Laboratories, Albuquerque, NM 87185, 1991

16 Kee R J, Rupley F M, Miller J A. CHEMKIN-II: a FORTRAN package for the analysis of gas-phase chemical kinetics. Sandia National Laboratory Report SAND89-8009B, 1989

17 Hall J M, Rickard M J A, Petersen E L. Comparison of characteristic time diagnostics for ignition and oxidation of fuel/oxidizer mixtures behind reflected shock waves. Combust Sci Technol, 2005, 177: 455-483

18 Law C K. Combustion Physics. Cambridge: Cambridge University Press, 2006

19 Slack M W. Rate coefficient for $\mathrm{H}+\mathrm{O}_{2}+\mathrm{M}=\mathrm{HO}_{2}+\mathrm{M}$ evaluated from shock tube measurements of induction times. Combust Flame, 1977, 28: 241-249

20 Bhaskaran K A, Gupta M C, Just T H. Shock tube study of the effect of unsymmetric dimethyl hydrazine on the ignition characteristics of hydrogen-air mixtures. Combust Flame, 1973, 21: 45-48

Open Access This article is distributed under the terms of the Creative Commons Attribution License which permits any use, distribution, and reproduction in any medium, provided the original author(s) and source are credited. 